\title{
Quand suspecter et comment diagnostiquer le trouble dissociatif de l'identité
}

\author{
Colin A. Ross \\ L'institut Colin A. Ross pour le trauma psychologique, Richardson, Texas, États-Unis
}

\begin{abstract}
Un trouble dissociatif de l'identité (TDI) jusque-là non diagnostiqué peut exister chez des individus qui sont évalués en vue de l'EMDR (désensibilisation et retraitement par les mouvements oculaires). Un TDI jusque-là non diagnostiqué était présent chez 3,9\% des 1529 patients adultes hospitalisés en psychiatrie générale dans 10 études menées dans 6 pays différents. Cet article présente un cas de TDI probable qui n'a pas été détecté dans un rapport de cas publié et apporte des lignes directrices pour déterminer quand suspecter et comment diagnostiquer le TDI. De telles lignes directrices manquent à la formation de nombreux professionnels en santé mentale.
\end{abstract}

Mots-clés : trouble dissociatif de l'identité ; diagnostic ; EMDR (désensibilisation et retraitement par les mouvements oculaires)

D ans 10 études menées dans 6 pays, le trouble dissociatif de l'identité (TDI) affectait 3,9\% parmi 1529 patients adultes hospitalisés en psychiatrie (Ross, Duffy, \& Ellason, 2002). Il s'agissait d'individus qui n'avaient pas reçu de diagnostic antérieur de TDI, n'avaient jamais reçu de traitement pour cela et ne prétendaient pas l'avoir. La procédure dans ces études, menées au Canada, aux États-Unis, en Turquie, en Norvège, en Suisse et en Allemagne, était d'évaluer les patients hospitalisés à l'aide de l'échelle des expériences dissociatives (Dissociative Experiences Scale [DES] ; Bernstein \& Putnam, 1986) puis de rencontrer en entretien ceux qui avaient obtenu un score élevé, à l'aide de l'un des deux entretiens structurés suivants : le Dissociative Disorders Interview Schedule (DDIS ; Ross, 1997 ; Ross \& Halpern, 2009) ou l'entretien clinique structuré du DSM-IV (Diagnostic and Statistical Manual of Mental Disorders, 4 éd.) pour les troubles dissociatifs (SCID-D ; Steinberg, 1995). À partir de cette épidémiologie, le TDI devrait faire partie du diagnostic différentiel pour de nombreux patients psychiatriques, y compris des patients évalués en vue de la pratique de l'EMDR (désensibilisation et retrai- tement par les mouvements oculaires) comme Shapiro $(1995,2001)$ le préconisait.

La littérature récente a abordé le besoin d'évaluer la présence d'un TDI ou d'autres troubles dissociatifs complexes dans la pratique de l'EMDR (Fine et coll., 2001 ; Gomez, 2012 ; Gonzalez \& Mosquera, 2012 ; Paulsen, 2009 ; Ross, 2012). Le niveau d'intérêt actuel envers le trauma complexe, la dissociation et l'EMDR est reflété par le nombre de présentations à ce sujet lors du dernier congrès de l'association internationale d'EMDR (EMDRIA ; Goldberg, 2014 ; Gomez, 2014 ; Korn \& Leeds, 2002 ; Leeds \& Mosquera, 2014 ; Madere, 2014 ; Ross, 2014). Que le protocole standard de l'EMDR soit suffisant pour le traitement du TDI n’a été ni confirmé ni infirmé par des essais contrôlés randomisés. Néanmoins, on retrouve un consensus voulant que des modifications soient requises, à tout le moins pour le recueil de l'histoire et la préparation (Fine \& Berkowitz, 2001 ; Forgash \& Copeley, 2008 ; Gomez, 2012 ; Gonzalez \& Mosquera, 2012 ; Leeds \& Mosquera, 2014 ; Paulsen, 1995, 2008, 2009 ; Twombly, 2000 ; Young, 1994). Il faudra peut-être travailler sur la stabilisation du système, l'ancrage, la communication

This article originally appeared as Ross, C. A. (2015). When to Suspect and How to Diagnose Dissociative Identity Disorder. Journal of EMDR Practice and Research, 9(2), 114-120. Translated by Jenny Ann Rydberg. 
et la coopération interpersonnalités, la co-conscience et l'orientation des personnalités alters au corps et au présent, par exemple.

Bien qu'il existe une littérature clinique sur l'EMDR dans le cas des troubles dissociatifs complexes, il n'existe pas à ce jour de protocole standard validé pour ces troubles. Les questions qui devront être soulevées dans le développement d'un tel protocole comprennent : les techniques de fractionnement (Kluft, 2013) sont-elles nécessaires à la sélection des cibles et à la préparation à la désensibilisation, la communication avec les personnalités devrait-elle être directe et/ou indirecte au cours de la préparation et de la désensibilisation, et quelles sont les techniques de traitement qui devraient être importées de la littérature sur le TDI ? La question qui demeure toujours sans réponse est de savoir si un protocole EMDR modifié est requis pour le TDI : si tel est le cas, une fois validé par des essais contrôlés, ce protocole pourrait alors devenir un protocole standard pour le TDI.

Étant donné les considérations susmentionnées, quand faudrait-il suspecter un TDI et comment en poser le diagnostic ? Cet article apporte des réponses à ces deux questions. La littérature récente sur les désaccords concernant la validité et la fiabilité du TDI ne constituent pas le point de mire ici (Boysen $\&$ VanBergen, 2013a, 2013b; Brand \& Loewenstein, 2014; Brand, Loewenstein \& Spiegel, 2013a, 2013b, 2014 ; Dalenberg et coll., 2012 ; Dorahy et coll., 2014 ; Lynn et coll., 2012 ; Martínez-Taboas, Dorahy, Sar, Middleton \& Krüger, 2013 ; Paris, 2012, 2013 ; Ross, 2013 ; voir

\section{TABLEAU 1. Idées fausses fréquentes sur le trouble dissociatif de l'identité (TDI)}

\begin{tabular}{lc}
\hline Le mythe & Les faits \\
\hline Rare. & $\begin{array}{c}\text { Un TDI non diagnostiqué affec- } \\
\text { tait 3,9\% des 1 } 529 \text { patients } \\
\text { hospitalisés en psychiatrie dans } \\
\text { six études dans 10 pays. }\end{array}$ \\
$\begin{array}{c}\text { Toujours iatrogène. } \\
\text { Peut être diagnostiqué sur la base } \\
\text { de symptômes préexistants. } \\
\text { diagnostiqué de } \\
\text { manière fiable. }\end{array}$ & $\begin{array}{c}\text { La fiabilité est bonne - valeurs de } \\
\text { kappa supérieures à 0,70. }\end{array}$ \\
$\begin{array}{c}\text { Le diagnostic } \\
\text { conduit à une } \\
\text { détérioration. }\end{array}$ & $\begin{array}{c}\text { Une littérature prospective non } \\
\text { contrôlée sur les résultats du } \\
\text { traitement démontre un bon } \\
\text { pronostic dans de nombreux cas. }\end{array}$ \\
$\begin{array}{c}\text { Pas de traitement } \\
\text { spécifié ou de soins } \\
\text { habituels. }\end{array}$ & $\begin{array}{c}\text { Le traitement est bien décrit dans } \\
\text { de nombreuses sources. }\end{array}$
\end{tabular}

tableau 1). En ce qui concerne les lignes directrices et les soins habituels concernant le traitement du TDI, le lecteur est orienté vers plusieurs sources (Brand \& Loewenstein, 2014 ; Chu et coll., 2004 ; Ross, 1997 ; Silberg et coll., 2011).

En ce qui concerne le TDI : (a) son diagnostic devrait se fonder sur des symptômes préexistants, dérivés du recueil direct de l'histoire et, lorsque possible, $\mathrm{du}$ recueil de renseignements auprès de proches; (b) un trouble factice et la simulation doivent être envisagés dans des contextes d'expertise et à chaque occasion où il existe un bénéfice secondaire potentiel ; (c) les symptômes du TDI, y compris l'existence de personnalités alters, sont les symptômes d'un trouble mental, pas des faits à prendre au sens littéral au sujet de la personne ; et (d) le fait d'avoir un TDI n'exonère pas automatiquement une personne de toute responsabilité morale, personnelle, éthique ou légale concernant ses actions.

\section{Critères diagnostiques du DSM-5 pour le trouble dissociatif de l'identité}

Le TDI est inclus dans la section des troubles dissociatifs de la cinquième édition du DSM (DSM-5 ; American Psychiatric Association, 2013). Les critères du DSM-5 sont inclus dans le DDIS (Ross, 1997 ; Ross \& Halpern, 2009) comme suit :

\section{Trouble dissociatif de l'identité}

127. Avez-vous déjà eu le sentiment qu'il y a deux états de personnalité distincts ou plus en vous, ce qui pourrait se décrire dans certaines cultures comme l'expérience d'être possédé ? Les états de personnalité causent une perturbation de votre sens de soi et s'accompagne de perturbations des émotions, des comportements, de la conscience, de la mémoire, des perceptions, de la pensée ou des sensations.

$$
\text { Oui }=1 \quad \text { Non }=2 \quad \text { Incertain }(e)=3
$$

128. Avez-vous déjà fait l'expérience d'une incapacité à vous rappeler des renseignements personnels importants ou des événements traumatiques qui soit d'une ampleur trop importante pour s'expliquer par une tendance à l'oubli ordinaire?

$$
\text { Oui }=1 \quad \text { Non }=2 \quad \text { Incertain }(e)=3
$$

129. Les symptômes ont-ils causé une détresse importante ou une détérioration de votre fonctionnement social, professionnel ou autre ?

$$
\text { Oui }=1 \quad \text { Non }=2 \quad \text { Incertain }(e)=3
$$


130. Le problème des différentes identités ou personnalités est-il causé par une prise d'alcool, de médicaments ou de substances illicites (p. ex. " trou noir » ou " black-out » alcoolique) ou une affection médicale générale ?

Oui $=1 \quad$ Non $=2 \quad$ Incertain $(\mathrm{e})=3 \quad[\quad]$

\section{Quand suspecter un trouble dissociatif de l'identité}

Bien que le TDI soit plus souvent diagnostiqué chez les femmes, des études montrent que le ratio femmeshommes du TDI non diagnostiqué ne dépasse pas 2:1 (Dorahy et coll., 2014 ; Ross, 1997 ; Ross et coll., 2002); le sexe n'est donc pas un facteur de risque important en soi. Les manifestations typiques d'un TDI jusquelà non diagnostiqué sont fournies dans le tableau 2 et dans l'étude de cas suivante.

\section{Exemple de cas}

L'étude de cas suivante provenant de la revue Current Psychiatry (Francois, Agakar \& Kotbi, 2012) contient de nombreux éléments d'un TDI non encore diagnostiqué :

Mademoiselle T., âgée de 20 ans, est accompagnée aux urgences par son père car elle refuse de boire et de manger, est incapable de fonctionner à la maison, reste au lit toute la journée et ne s'occupe pas de ses activités de la vie quotidienne (AVQ).
Aux urgences, elle ne coopère pas initialement ni ne parle, puis elle devient soudain agitée et présente un épisode épileptiforme, son torse étant traversé de mouvements saccadés, suivis de mouvements aléatoires et asymétriques de ses bras et de ses jambes, les yeux fermés, avec des pleurs, de l'écume buccale, des gémissements, mais sans aucune réaction vis-à-vis de son environnement. Cet épisode dure plus de cinq minutes.

Dans l'unité psychiatrique, Mademoiselle T. est d'abord irritable et désorganisée, avec une prise alimentaire faible et une régression comportementale ; on la trouve souvent en position fotale, en pleurs et s'exprimant de manière enfantine.

Son psychiatre en consultation externe décrit une histoire de maltraitances physiques et sexuelles à partir de l'âge de sept ans. Quand elle a neuf ans, après le décès de sa mère d'un cancer du sein, Mademoiselle $T$. et ses frères et sœurs sont placés dans un foyer où elle subit des maltraitances physiques de la part du personnel. Sa garde a été confiée aux services sociaux jusqu’à ses dix-huit ans.

Le père de Mademoiselle $T$. dit que sa fille a été hospitalisée plusieurs fois en raison d'épisodes caractérisés par des mouvements de va-et-vient du bassin, des bégaiements et un pseudosommeil ...

Nous avons également envisagé un trouble de conversion et un trouble dissociatif ...

\section{TABLEAU 2. Quand suspecter un trouble dissociatif de l'identité}

Traumatisation

Trouble de la personnalité borderline (TPB)

Voix

Absences

Passages d'un état à un autre (switching) Diagnostics antérieurs
Une traumatisation sévère considérable dans l'enfance.

N'a pas besoin d'être corroborée.

N'a pas besoin d'inclure des violences sexuelles.

Diagnostic antérieur de TPB.

Critères du TPB actuellement satisfaits ou infracliniques.

La dépression et l'ESPT comorbides sont également fréquents.

Hallucinations auditives - généralement chroniques.

Les voix peuvent avoir des noms et des âges connus.

Les voix répondent souvent au critère A du DSM-IV pour la schizophrénie.

Périodes délimitées de temps manquant, d’une durée de plusieurs minutes, heures ou jours, non causées par des médicaments, des drogues, l'alcool ou une affection médicale connue

Changements soudains de l'état comportemental

Antécédents de contacts nombreux et fréquents avec le système de santé mentale Nombreux diagnostics antérieures - comprenant souvent le TPB, le trouble bipolaire, la schizophrénie, le trouble schizoaffectif, l'ESPT et la toxicomanie

Note. ESPT $=$ état de stress post-traumatique $;$ DSM-IV = Diagnostic and Statistical Manual of Mental Disorders, $4^{\text {th }}$ ed. (manuel diagnostique et statistique des troubles mentaux, $4^{\mathrm{e}}$ edition). 
Le diagnostic de crises pseudoépileptiques posé par les auteurs dans ce cas est techniquement correct mais ne rend pas compte du tableau clinique complet. Bien qu'un trouble dissociatif ait été envisagé, rien n'indique que le TDI ait été systématiquement exclu. Il serait intéressant d'interviewer Mademoiselle T. à l'aide de l'EED (DES ; Bernstein \& Putnam, 1986), un questionnaire d'autoévaluation largement utilisé, et du DDIS (Ross, 1997 ; Ross \& Halpern, 2009), et qu'elle soit ensuite évaluée par un clinicien compétent dans le diagnostic du TDI. L'EED et le DDIS sont des documents appartenant au domaine public et possèdent une fiabilité et un validité établies (Ross, 1997 ; Ross \& Halpern, 2009). Les deux outils figurent dans le Manuel des outils de mesure psychiatriques de la Société américaine de psychiatrie (Rush, First $\&$ Blacke, 2007).

Plutôt que de simples crises pseudoépileptiques, les épisodes épileptiformes de Mademoiselle T. pouvaient très correspondre à des abréactions de viols dans l'enfance de la part d'une personnalité alter infantile. Le TDI expliquerait les changements soudains d'états comportementaux, les pleurs, la voix enfantine et le pseudosommeil. Les antécédents de violences sexuelles expliqueraient le composant des mouvements de va-et-vient du bassin pendant les crises pseudoépileptiques. Comment Francois et coll. (2012) auraient-ils pu investiguer le diagnostic de TDI chez Mademoiselle T. au cours d'un entretien clinique?

\section{Comment diagnostiquer le trouble dissociatif de l'identité}

La procédure et l'approche destinées à diagnostiquer le TDI sont les mêmes que pour tout autre trouble mental. Le TDI peut être suspecté mais ne peut être diagnostiqué chez une personne psychotique dont la symptomatologie est florissante, avec un trouble sévère de la pensée, ni chez un individu intoxiqué, délirant, non coopératif, muet ou autrement incapable de participer à un entretien psychiatrique. L'évaluation comprend tous les éléments classiques d'une évaluation psychiatrique, dont le recueil de renseignements complémentaires auprès de tierces personnes et un examen de l'état mental. Toutefois, des questions supplémentaires, ne faisant pas partie de la plupart des pratiques cliniques (voir tableau 3), sont nécessaires.

Deux études (Putnam, Guroff, Silberman, Barban \& Post, 1989 ; Ross, Norton \& Wozney, 1989) ont démontré que, dans les années 1980, les patients avec une personnalité multiple passaient en moyenne un peu moins de sept ans dans le système de santé mentale avant que leur trouble dissociatif soit diagnostiqué. Rien n’indique que ce délai se soit réduit depuis, malgré l'émergence d'une littérature conséquente sur le TDI au cours des vingt-cinq années qui ont suivi. Bien que quelques cas de TDI puissent être difficiles à diagnostiquer, le délai s'explique essentiellement par l'omission d'un recueil d'antécédents suffisant et spécifique.

Quand les questions nécessaires sont posées, le TDI peut souvent être diagnostiqué au cours d'un seul entretien. Toutefois, comme pour tout trouble, le diagnostic peut demander plus de temps dans certains cas et peut nécessiter le développement d'une alliance thérapeutique. Généralement, dans de tels cas, le diagnostic provisoire de trouble dissociatif non spécifié du DSM-5 peut être posé (American Psychiatric Association, 2013). Des questions précises concernant " d'autres personnes à l'intérieur de vous » devraient être posées en dernière partie de l'entretien, après l'exploration des autres symptômes du tableau 3.

Comme lors de tout entretien, on commence par des questions ouvertes pour arriver à des questions plus fermées. Les personnes atteintes d'un TDI décrivent rarement leurs symptômes de manière spontanée. Il peut y avoir de longues périodes de rémission pendant lesquelles les personnalités alter étaient latentes ou dormantes, et donc aucun symptôme du tableau 3 n'était alors présent. Généralement, toutefois, ceci n'est pas le cas lorsque la personne est en crise et a été admise par le système de santé mentale. Si on présume l'existence d'autres "personnes " intérieures qui prennent le contrôle du corps, plus la présence d'amnésie, les symptômes du tableau 3 en découlent logiquement. Il existe souvent une amnésie étendue pour l'enfance, par exemple, une incapacité à se rappeler quoi que ce soit avant l'âge de 13 ans, ou une amnésie pour une période de temps donnée, par exemple entre huit et douze ans lorsqu'un beau-père vivait à la maison. Dans d'autres cas, l'amnésie infantile est parcellaire et plus difficile à distinguer d'un oubli normal. La caractéristique principale du TDI demeure cependant des absences ou trous de mémoire distincts en l'absence de drogues, de médicaments ou d'alcool. Dans quelques rares cas, ceci peut être difficile à distinguer d'un oubli normal ou de la tendance à être dans la lune, mais dans la plupart des cas, la distinction est facile à établir.

Beaucoup de symptômes du TDI existent sur un continuum. Les symptômes du TDI peuvent être difficiles à distinguer d'un état normal ou de troubles dissociatifs moins sévères lors de l'évaluation initiale. Cependant, quand une personne décrit des voix avec un nom, un âge et un sexe défini parlant dans sa tête, 
Ouvertes

Absences/trous de mémoire

Sortie des absences

Événements oubliés

Des inconnus qui vous connaissent

Objets manquants/présents

Écriture différente

Voix

D’autres « personnes »

Renseignements de tierces personnes
Des problèmes de mémoire?

Des périodes de temps manquantes - se poursuivant pendant des minutes, des heures ou des jours

Début et fin abruptes

Récurrentes

Pas de drogue ou d'alcool

Revenir à soi dans un lieu différent ; ne pas savoir comment on y est arrivé

Des personnes vous parlent de choses que vous avez faites mais dont vous ne vous souvenez pas - décrivent des comportements très différents de votre habitude

Des personnes que vous ne reconnaissez pas semblent vous connaître peuvent vous appeler par un nom différent

Des objets peuvent disparaître ou apparaître sans que vous puissiez en rendre compte - pas de simples oublis mineurs - p. ex. trouver des vêtements récemment achetés dans votre armoire, d'un style que vous ne porteriez jamais

Trouver des écrits rédigés dans une écriture différente et que vous ne vous rappelez pas avoir faits : peuvent être enfantins, colériques, parler de vous

Entendre des voix - qui peuvent vous parler ou parler entre elles ; il peut y en avoir plusieurs ; elles peuvent avoir un âge, un nom ou un genre

Avez-vous le sentiment qu'il pourrait y avoir une ou plusieurs autres personnes en vous ? Si oui, prennent-elles parfois le contrôle de votre corps ?

Des proches / amis peuvent décrire des changements soudains de comportement avec de l'amnésie, peuvent connaître des parties et leur nom, peuvent corroborer des antécédents traumatiques ou rapporter qu'ils ont été maltraités par les mêmes auteurs de violences le fait de se retrouver dans des lieux non familiers (par exemple, dans le lit d'un inconnu) au terme d'une période de deux jours sans aucun souvenir de ce qui s'est passé, des traumas sévères dans l'enfance et un sentiment qu'il existe d'autres personnes en elle, la probabilité d'un TDI est élevée.

Il n'y a pas de mystère quant à savoir pourquoi de nombreux cliniciens ne voient pas de cas de TDI. Ils n'interrogent pas les symptômes ni n'envisagent le TDI dans leurs diagnostics différentiels. Des questions systématiques telles que décrites dans cet article, ou le recours à l'EED et au DDIS devraient aisément permettre d'identifier des cas de TDI dans des échantillons de patients hospitalisés en psychiatrie dans la plupart des institutions. De manière similaire, des cas de TDI non diagnostiqués ne devraient pas être difficiles à trouver dans les pratiques de l'EMDR. Il s'agira de patients qui n'ont jamais reçu le diagnostic, n'ont jamais reçu de traitement pour ce trouble et ne le revendiquent pas. Il s'agit d'une hypothèse scientifique susceptible d'être testée qui a déjà été répliquée dans six pays différents (Ross et coll., 2002). On peut s'attendre à trouver un TDI jusque-là non diagnostiqué chez des patients évalués pour une indication de l'EMDR car ils possèdent souvent des antécédents traumatiques.

En général, le TDI sera plus facilement diagnostiqué dans un service d'urgences ou dans le cadre d'une hospitalisation parce que l'individu est en crise, déstabilisé, moins organisé, moins ancré et davantage susceptible d'avoir eu des symptômes florissants dans un passé récent. Aussi, la contenance, le soutien et la structure du cadre hospitalier facilite la prise de risque associée à la divulgation des symptômes. Dans un contexte ambulatoire, comme une pratique libérale de l'EMDR, par contre, il est probable que les cas demanderont plus de temps pour être diagnostiqués. Les patients suivis en ambulatoire sont généralement plus stables, mieux organisés et plus fonctionnels ; c'est pourquoi ils sont moins susceptibles de connaître des moments d'absence ou "trous de mémoire », des voix ou d'autres symptômes de TDI. Le diagnostic peut nécessiter plus de temps parce que le système de la personnalité TDI peut être plus latent ou éteint. 
De plus, du temps supplémentaire peut être requis pour développer une alliance thérapeutique suffisante pour que la personne prenne le risque de divulguer ses symptômes. Quel que soit le contexte, toutefois, le même entretien clinique, appuyé par l'EED et le DDIS, constitue un moyen efficace d'évaluer la présence d'un TDI ou d'un autre trouble dissociatif complexe chronique chez un individu.

En conclusion, si des essais randomisés contrôlés de l'EMDR pour le TDI doivent être réalisés à l'avenir, alors un protocole standard sera nécessaire. La question de savoir si le protocole EMDR standard pour le traitement du TDI devrait comporter des modifications du protocole standard existant dépend des résultats des études contrôlées. Quel que soit le protocole, toutefois, une évaluation systématique pour déceler un TDI jusque-là non diagnostiqué sera requis. Des lignes directrices pour l'évaluation clinique d'un TDI sont présentées dans cet article et d'autres ressources citées ici ; l'ajout de l'EED et du DDIS à l'entretien clinique est recommandé.

\section{Bibliographie}

American Psychiatric Association. (2013). Diagnostic and statistical manual of mental disorders ( $5^{\mathrm{e}}$ éd.). Washington, DC : Auteur.

Bernstein, E. M., \& Putnam, F. W. (1986). Development, reliability and validity of a dissociation scale. Journal of Nervous and Mental Disease, 174, 727-735.

Boysen, G. A., \& VanBergen, A. (2013a). A review of dissociative identity disorder research: Response to critics. Journal of Nervous and Mental Disease, 201, 440-443.

Boysen, G. A., \& VanBergen, A. (2013b). A review of published research on adult dissociative identity disorder: 2000-2010. Journal of Nervous and Mental Disease, 201, 5-11.

Brand, B. L., \& Loewenstein, R. J. (2014). Does phasic trauma treatment make patients with dissociative identity disorder treatment more dissociative? Journal of Trauma \& Dissociation, 15, 52-65.

Brand, B. L., Loewenstein, R. J., \& Spiegel, D. (2013a). Disinformation about dissociation: Dr. Joel Paris's notions about dissociative identity disorder. Journal of Nervous and Mental Disease, 201, 354-356.

Brand, B. L., Loewenstein, R. J., \& Spiegel, D. (2013b). Patients with DID are found and researched more widely than Boysen and VanBergen recognized. Journal of Nervous and Mental Disease, 201, 440.

Brand, B. L., Loewenstein, R. J., \& Spiegel, D. (2014). Dispelling myths about dissociative identity disorder treatment: An empirically based approach. Psychiatry, 77, 169-89.

Chu, J., Dell, P. F., Van der Hart, O., Cardena, E., Barach, P. M., Somer, E., . . Twombly, J. (2011). Guidelines for treating dissociative identity disorder in adults, third revision. Journal of Trauma \& Dissociation, 12, 115-187.

Dalenberg, C. J., Brand, B. L., Gleaves, D. H., Dorahy, M. J., Loewenstein, R. J., Cardeña, E., . . . Spiegel, D. (2012). Evaluation of the evidence for the trauma and fantasy models of dissociation. Psychological Bulletin, 138, $550-588$

Dorahy, M. J., Brand, B. L., Sar, V., Krüger, C., Stavropoulos, P., Martínez-Taboas, A., ... Middleton, W. (2014). Dissociative identity disorder: An empirical review. Australia and New Zealand Journal of Psychiatry, 48, 402-417.

Fine, C. G., \& Berkowitz, A. S. (2001). The wreathing protocol: The imbrication of hypnosis and EMDR in the treatment of dissociative identity disorder and other dissociative responses. American Journal of Clinical Hypnosis, 43, 275-290.

Fine, C. G., Paulsen, S., Rouanzoin, C., Luber, M., Puk, G., \& Young, W. (2001). A general guide to the use of EMDR in the dissociative disorders. In F. Shapiro (Ed.), Eye movement desensitization and reprocessing (EMDR): Basic principles, practices and procedures (2 éd., p. 365-369). New York, NY : Guilford Press.

Forgash, C., \& Copeley, M. (2008). Healing the heart of trauma and dissociation with EMDR and ego state therapy. New York, NY: Springer Publishing.

Francois, S., Agakar, S., \& Kotbi, N. (2012). Epilepsy or something else? Current Psychiatry, 11, 46-50.

Goldberg, A. (2014, septembre). Relational affect regulation for complex trauma survivors. Présentation lors du $20^{\mathrm{e}}$ congrès annuel de l'EMDR International Association, Denver, CO.

Gomez, A. M. (2012). EMDR therapy and adjunct approaches with children: Complex trauma, attachment, and dissociation. New York, NY : Springer Publishing.

Gomez, A. M. (2014, September). There and back again: The intricate and fascinating journey of helping children with complex trauma using EMDR therapy. Présentation lors du $20^{\mathrm{e}}$ congrès annuel de l'EMDR International Association, Denver, CO.

Gonzalez, A., \& Mosquera, D. (2012). EMDR and dissociation: The progressive approach. Madrid, Spain: Ediciones Pléyades, S. A.

Kluft, R. P. (2013). Shelter from the storm: Processing the traumatic memories of DID/DDNOS patients with the fractionated abreaction technique. North Charlestown, SC : CreateSpace Independent Publishing Platform.

Korn, D. L., \& Leeds, A. M. (2002). Preliminary evidence of efficacy for EMDR resource development and installation in the stabilization phase of treatment of complex posttraumatic stress disorder. Journal of Clinical Psychology, 58, 1465-1487.

Leeds, A., \& Mosquera, D. (2014, septembre). History taking and preparation for EMDR therapy in complex trauma. Présentation lors du $20^{\mathrm{e}}$ congrès annuel de l'EMDR International Association, Denver, CO.

Lynn S. J., Lilienfeld, S. O., Merckelbach, H., Giesbrecht, T., McNally, R. J., Loftus, E. F., . . Malaktaris, A. (2014). 
The trauma model of dissociation: Inconvenient truths and stubborn fictions. Comment on Dalenberg et al. (2012). Psychological Bulletin, 140, 896-910.

Madere, J. (2014, September). Comparing ACES \& DES scores: Implications for assessment of diverse populations. Présentation lors du 20e congrès annuel de l'EMDR International Association, Denver, CO.

Martínez-Taboas, A., Dorahy, M., Sar, V., Middleton, W., \& Krüger, C. (2013). Growing not dwindling: International research on the worldwide phenomenon of dissociative disorders. Journal of Nervous and Mental Disease, 201, 353-354.

Paris, J. (2012). The rise and fall of dissociative identity disorder. Journal of Nervous and Mental Disease, 200, 1076-1079.

Paris, J. (2013). Response to dissociative identity disorder letters from Martínez-Taboas et al. and Brand et al. Journal of Nervous and Mental Disease, 201, 356-357.

Paulsen, S. (1995). Eye movement desensitization and reprocessing: Its cautious use in the dissociative disorders. Dissociation, 8, 32-44.

Paulsen, S. (2008). Treating dissociative identity disorder with EMDR, ego state therapy, and adjunct approaches. In C. Forgash \& M. Copeley (Eds.), Healing the heart of trauma and dissociation with EMDR and ego state therapy (p. 141-180). New York, NY : Springer Publishing.

Paulsen. S. (2009). Looking through the eyes of trauma and dissociation. An illustrated guide for EMDR therapists and clients. Charleston, SC: BookSurge.

Putnam, F. W., Guroff, J. J., Silberman, E. K., Barban, L., \& Post, R. M. (1989). The clinical phenomenology of multiple personality disorder: Review of 100 recent cases. Journal of Clinical Psychiatry, 45, 172-175.

Ross, C. A. (1997). Dissociative identity disorder: Diagnosis, clinical features, and treatment of multiple personality ( $2^{\mathrm{e}}$ éd.). New York, NY : John Wiley.

Ross. C. A. (2012). EMDR is based on a trauma-dissociation model of mental disorders. Revista Iberoamericana de Psicotraumatologia y Disociacion, 3, 1-17.

Ross, C. A. (2013). Commentary: The rise and persistence of dissociative identity disorder. Journal of Trauma \& Dissociation, 14, 584-588.
Ross, C. A. (2014, September). How to diagnose dissociative disorders. Présentation lors du 20e congrès annuel de l'EMDR International Association, Denver, CO.

Ross, C. A., Duffy, C. M. M., \& Ellason, J. W. (2002). Prevalence, reliability, and validity of dissociative disorders in an inpatient setting. Journal of Trauma \& Dissociation, 3, 7-17.

Ross, C. A., \& Halpern, N. (2009). Trauma model therapy: A treatment approach for trauma, dissociation, and complex comorbidity. Richardson, TX : Manitou Communications.

Ross, C. A., Norton, G. R., \& Wozney, K. (1989). Multiple personality disorder: An analysis of 236 cases. Canadian Journal of Psychiatry, 34, 413-418.

Rush, A. J., First, M. B., \& Blacke, D. (2007). Handbook of psychiatric measures ( ${ }^{\mathrm{e}}$ éd.). Washington, DC : American Psychiatric Publishing.

Shapiro, F. (1995). Eye movement desensitization and reprocessing (EMDR): Basic principles, protocols, and procedures. New York, NY : Guilford Press.

Shapiro, F. (2001). Eye movement desensitization and reprocessing (EMDR): Basic principles, protocols, and procedures (2e éd.). New York, NY : Guilford Press.

Silberg, J., Waters, F., Nemzer, E., McIntee, J., Wieland, S., Grimminck, E., ... Emsond, E. (2004). Guidelines for the evaluation and treatment of dissociative symptoms in children and adolescents. Journal of Trauma \& Dissociation, 5, 119-150.

Steinberg, M. (1995). Handbook for the assessment of dissociation: A clinical guide. Washington, DC : American Psychiatric Press.

Twombly, J. H. (2000). Incorporating EMDR and EMDR adaptations into the treatment of clients with dissociative identity disorder. Journal of Trauma and Dissociation, 1, 61-81.

Young, W. C. (1994). EMDR treatment of phobic symptoms in multiple personality disorder. Dissociation, 7, 129-133.

Merci d'adresser toute correspondence concernant cet article à Colin A. Ross, MD, President, The Colin A. Ross Institute for Psychological Trauma, 1701 Gateway \#349, Richardson, TX 75080, États-Unis. Courriel : rossinst@ rossinst.com 